\title{
Increased cardiac electrical instability concomitant with pacing induced repolarisation abnormalities
}

\author{
PAOLO DELla BELLA, SERGIO GRAZI, CARLO M CIPOLLA, \\ FRANCO FABBIOCCHI, ANDREA RIMONDINI, PAOLO SGANZERLA, \\ MAURIZIO D GUAZZI \\ From the Istituto di Cardiologia, Istituto di Ricerche Cardiovascolari "G Sisini", Centro Ricerche \\ Cardiovascolari del Consiglio Nazionale delle Ricerche, Fondazione "Italo Monzino", University of Milan, \\ Italy
}

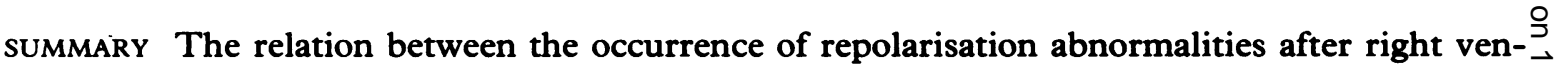
tricular pacing and spontaneous arrhythmias was investigated in 16 patients in whom the sick sinus syndrome was suspected. All patients had normal QRS complexes and $T$ waves in the음 electrocardiogram before pacing and required atrial stimulation and His bundle recording for diagnostic purposes. Patients were randomised into a study group or a control group. In the eight patients in the study group right ventricular pacing was performed for 12 hours, and was followed $\vec{\oplus}$ by inversion of the T wave in surface leads II, III, aVF, and V2-V5 and lengthening of the QTc. ${ }^{-}$ interval. The frequency and complexity of ventricular arrhythmias increased after pacing in six patients who had ventricular extrasystoles in the baseline Holter recording. As the configuration of the $T$ wave became normal the frequency of ventricular extrasystoles returned to baseline values. In the control group of eight patients ventricular pacing was not performed after the $\frac{\mathbb{Q}}{\mathbb{D}}$ electrophysiological study and no changes were seen in $T$ wave configuration and in the frequency of spontaneous arrhythmias.

These results suggest that the post-pacing repolarisation abnormalities reflect abnormal electrical properties of the ventricle and that in some cases they lead to increased electrical instability.

Abnormal ventricular depolarisation causes abnormal repolarisation which is manifested as $T$ wave abnormalities. Transient abnormal repolarisation may be seen after the recovery of normal intraventricular conduction in patients with intermittent left bundle branch block, ${ }^{1}$ temporary right ventricular pacing, ${ }^{23}$ or intermittent ventricular preexcitation. ${ }^{45}$ Rosenbaum et $a l^{6}$ described the time course of $T$ wave inversion after ventricular pacing and suggested that electrotonic modulation is responsible for the altered repolarisation that follows anomalous activation and that "accumulation" and "memory" of the new properties occur; these were said to account for the persistence of the repolarisation abnormalities.

Requests for reprints to Dr Paolo Della Bella, Istituto di Cardiologia, Via Bonfadini 214, 20138 Milan, Italy.

Accepted for publication 30 September 1986
The clinical significance of these post-pacing abnormalities of repolarisation is still controversial. $\dot{\alpha}$ A possible relation to myocardial ischaemia was proposed initially; however, these abnormalities also 0 occur in the absence of coronary artery disease. ${ }^{6}$ Electrical stimulation of the myocardium induces $\frac{7}{0}$ profound alterations of the electrical properties within the stimulated area, ${ }^{78}$ and unequal recovery $O$ of the cardiac muscle increases vulnerability to $N$ arrhythmias. ${ }^{910}$

We have investigated whether the frequency of $O$ spontaneous arrhythmias changed during the repolarisation abnormalities caused by a period of right ventricular pacing.

\section{Patients and methods}

PATIENTS

We studied 16 patients (nine men and seven women; 
mean age 57 years, range $43-68$ years). They were randomised into a study or a control group (eight patients in each group). In all of them sinus node disease was suspected on the basis of repeated episodes of bradycardia and was the indication for an electrophysiological study. The electrocardiogram showed normal ventricular activation and repolarisation in all patients before the study. The axis of the QRS complex in the frontal plane lay between $0^{\circ}$ and $90^{\circ}$. Coronary artery disease was excluded on the basis of history, clinical observation, resting and exercise electrocardiogram, and 24 hour Holter recording. Coronary angiography was performed in seven patients and was normal in all. In 10 patients (six in the study group and four in the control group) a 24 hour Holter recording showed ventricular extrasystoles of variable frequency, which were not symptomatic.

All patients gave informed written consent to the study including the invasive procedure and the stimulation period after the diagnostic electrophysiological study.

\section{STIMULATION PROTOCOL}

The diagnostic electrophysiological study, including the His bundle recording, was performed in both groups.

After the diagnostic procedures had been completed in the patients in the study group a bipolar or tripolar electrode catheter was advanced to the apex of the right ventricle to a position with a stimulation threshold of between 0.5 and $1.0 \mathrm{~mA}$. A Medtronic programmable stimulator (model 5325) was used to deliver stimuli. Square wave pulses lasting $2 \mathrm{~ms}$ were given at twice diastolic threshold. The lowest possible pacing rate that ensured constant ventricular capture (between 70 and 90 beats $/ \mathrm{min}$ ) was used. Pacing was started at $8 \mathrm{pm}$ and was continued for 12 hours in all patients. The lower cardiac rate during the night permits ventricular pacing at low rates. In the control group electrode catheters were withdrawn after the electrophysiological study, and electrocardiographic recordings and Holter monitorings were performed in the same way as in the study group.

\section{ELECTROCARDIOGRAPHIC RECORDINGS}

Electrocardiograms were recorded at a paper speed of $100 \mathrm{~mm} / \mathrm{s}$ and high amplification $(50 \mathrm{~mm} / \mathrm{mV})$ by means of an optically isolated polygraph (Hewlett Packard 4578 A). Electrocardiograms were obtained before and immediately at the end of the pacing period during the return of spontaneous sinus rhythm and $1,2,4,6$, and 12 hours after the end of pacing. Further electrocardiograms were recorded every 12 hours until the pacing induced repolarisation abnormalities disappeared. We measured the ventricular gradient in the frontal plane from the QRS and ST-T areas in leads I, II, III, and in single precordial leads (V3 and V4). The values given are the mean of five complexes. Planimetry of the tracings was performed with a Kontron computer. The area from the beginning of the QRS to the $J$ point and that from the J point to the end of the $T$ wave were regarded as the $Q R S$ and $T$ areas. The mean electric axis and measured area of the ventricular gradient in the frontal plane were derived according to the rules described by Ashman and Byer. ${ }^{11}$ Values for the QT interval were obtained by averaging the values of five beats on the three standard leads. We used Bazett's formula to correct the QT interval for heart rate. ${ }^{12}$ Continuous 24 hour Holter recordings were obtained with an Avionics model 1445/B two channel recorder for $(a)$ the day before pacing, $(b)$ after the end of pacing, and $(c)$ when the repolarisation abnormalities had unequivocally disappeared. Tape recordings were analysed by the same staff, using a Del Mar Avionics Trendsetter model DCG VII Dynamic Electrocardioscanner. The following data were measured for each patient: the total number of heart beats in the 24 hours, the highest and the lowest heart rates per minute, the frequency of ventricular extrasystoles in 24 hours, or of "complex" ventricular arrhythmias (couplets, runs of ventricular tachycardia). All recordings were started at $9 \mathrm{am}$. In some patients we also used direct electrocardiographic recording (Hewlett Packard 4700 A Pagewriter) to assess the hourly frequency of ventricular extrasystoles throughout the observation period. In all patients the results were similar to those obtained from Holter recording.

\section{STATISTICAL ANALYSIS}

The values of RR and QTc interval in control conditions and during various periods of observation were compared by Student's $t$ test. A p value of $<0.05$ was regarded as significant. We used a Kontron computer, model Cardio 80, for statistical analysis.

\section{Results}

STUDY GROUP

Electrocardiographic measurements

The period of ventricular stimulation was followed by persistent repolarisation changes (fig 1 ). The following features were seen in all eight patients in the study group: (a) inversion of the T wave in leads II, III, and aVF, leading to a shift of the $T$ wave vector leftwards and upwards; $(b)$ significant leftward deviation of the ventricular gradient in the frontal plane; and $(c)$ decrease of the measured area. 


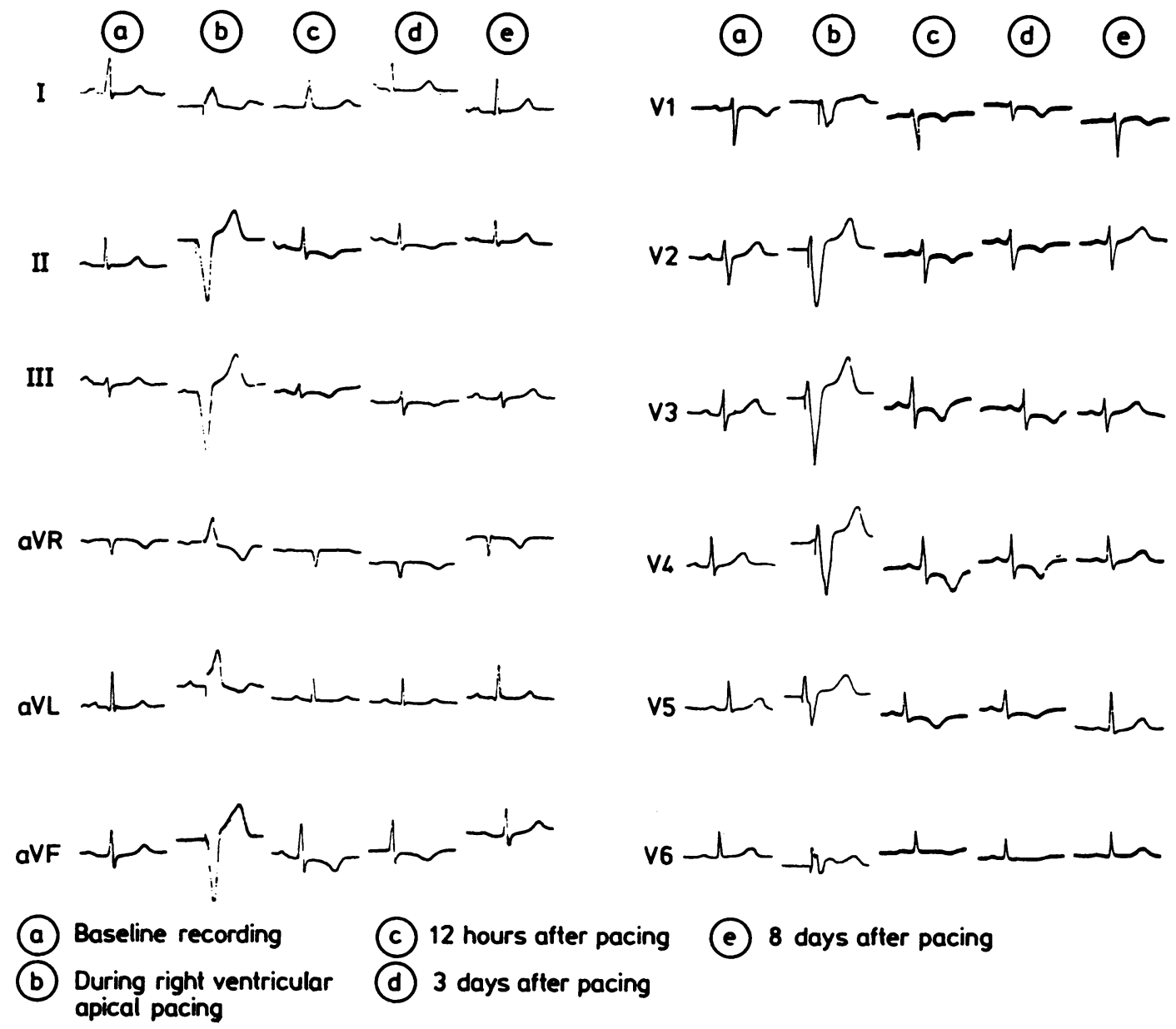

Fig 1 Typical pattern of $T$ wave changes on a 12 lead electrocardiogram before, during, and after right ventricular pacing in patient 1 . The polarity of the inverted $T$ wave is the same as that of the paced $Q R S$. Repolarisation had returned to normal several days after the end of right ventricular pacing.

The T wave from leads V2 to V5 was inverted in five patients, and in the remaining three it was flattened or reduced in amplitude in these leads. These changes were evident at the end of pacing and reached a maximum 2-12 hours after the end of pacing. Reversion of these abnormalities was slow, with restoration of the original values 5-22 days after the end of ventricular pacing (table 1). Figure 2 shows baseline and maximum values of the axis of the $T$ wave and of the ventricular gradient. After stimulation all patients also showed a transient lengthening of the QTc interval which was greatest 4-24 hours (mean 12 hours) after ventricular pacing. The mean QTc interval increased from 409.8 to $446.0 \mathrm{~ms}(\mathrm{p}<0.001)$ in the absence of significant variations in the $R R$ interval. The $Q T c$ interval val- ues returned to their original values within $\mathbf{4 8}$ hourso in all patients (table 2 ).

Table 1 Time at which $T$ wave inversion was greatest and at which the repolarisation changes returned to normal after right ventricular pacing

\begin{tabular}{|c|c|c|}
\hline Patient No & $\begin{array}{l}\text { Maximal } T \\
\text { inversion }(h)\end{array}$ & $\begin{array}{l}\text { Normal at } \\
\text { (days) }\end{array}$ \\
\hline $\begin{array}{l}1 \\
2 \\
3 \\
4 \\
5 \\
6 \\
7 \\
8\end{array}$ & $\begin{array}{r}4 \\
6 \\
7 \\
6 \\
8 \\
3 \\
12 \\
2\end{array}$ & $\begin{array}{r}22 \\
8 \\
5 \\
5 \\
8 \\
6 \\
5 \\
10\end{array}$ \\
\hline
\end{tabular}



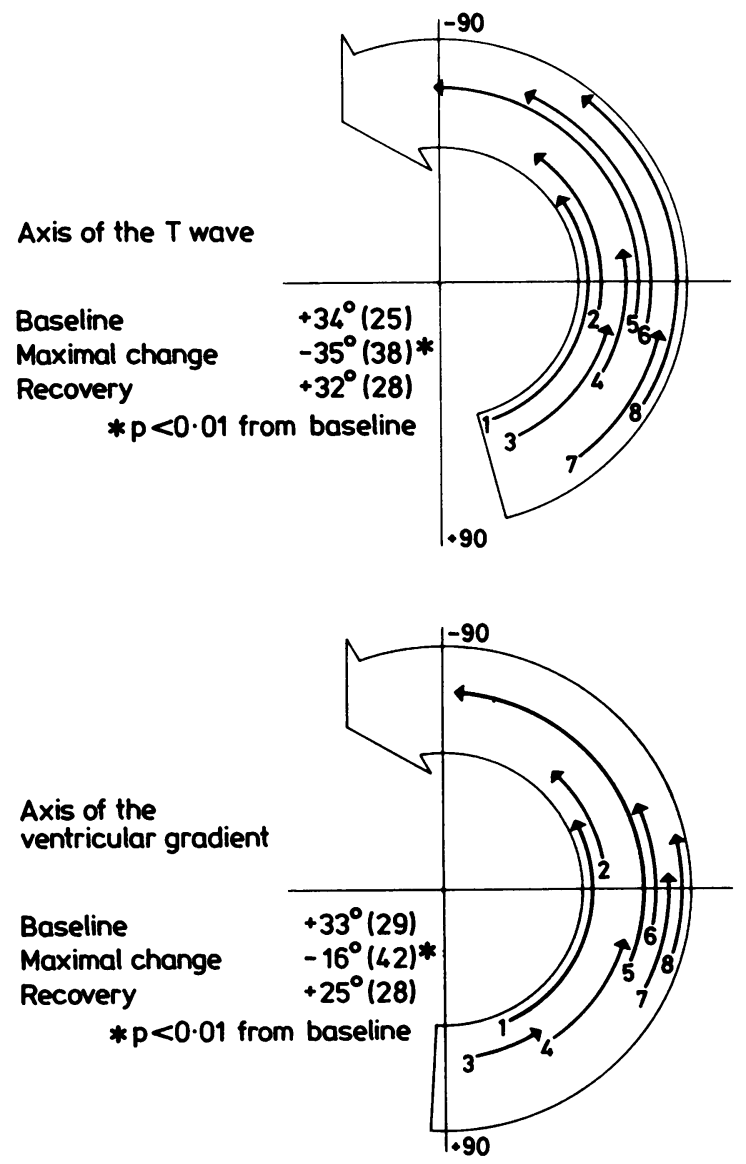

Fig 2 Range of $T$ wave axes and ventricular gradient distribution in patients in baseline conditions and at maximal shift after ventricular pacing. Mean (SD) values are shown.

Holter recordings (table 3 )

In each patient the total number of heart beats during the 24 hours was the same in all recordings, suggesting that the mean heart rate remained constant.
The values for the highest and lowest heart rates in both early and late post-pacing recordings resembled those in the baseline recording. Patients 4 and 7 , in whom no arrhythmias were seen in the baseline recording, had no spontaneous arrhythmias in the earlier (first 24 hours) and in the late (after disappearance of the repolarisation abnormalities) postpacing Holter monitoring. In patients $1,2,3,5,6$, and 8 , however, who had premature ventricular beats before pacing, there was a remarkable increase in the frequency of these arrhythmias in the 24 hours after pacing. Several complex ventricular arrhythmias were seen. In case 3 ventricular extrasystoles of two different configurations and two couplets were present in the baseline Holter recording, and soon after the end of pacing we saw ventricular extrasystoles with three distinct configurations and several short episodes ( 3 to 4 beats) of ventricular tachycardia. Tachycardia was not detectable in the third Holter recording. In patient 6 , who presented with single uniform ventricular extrasystoles in the baseline study, there were couplets and two short runs of ventricular tachycardia in the early postpacing recording. These arrhythmias were not present in the later recording.

Figure 3 shows the strict relation between changes in repolarisation (ventricular gradient, QTc value) after pacing and the frequency of ventricular extrasystoles in patient 1 . Repolarisation changes were most pronounced 12-24 hours after pacing, at the same time as the frequency of spontaneous arrhythmias increased. The frequency of these arrhythmias decreased as the pacing induced repolarisation changes returned to normal. In patients 2 and 8 the number of ventricular extrasystoles greatly increased in the 24 hours immediately after pacing and returned toward the baseline values during the last recording.

CONTROL GROUP

Analysis of the electrocardiograms showed no

Table $2 Q T c$ and RR intervals (ms) in the study group

\begin{tabular}{|c|c|c|c|c|c|c|}
\hline \multirow[b]{2}{*}{ Patient No } & \multicolumn{2}{|l|}{ Baseline } & \multicolumn{2}{|c|}{ Maximal change } & \multicolumn{2}{|l|}{ Recovery } \\
\hline & $Q T c$ & $\boldsymbol{R} R$ & $Q T c$ & $\boldsymbol{R} R$ & $Q T c$ & $\boldsymbol{R} R$ \\
\hline $\begin{array}{l}1 \\
2 \\
3 \\
4 \\
5 \\
6 \\
7 \\
8 \\
\text { Mean (SD) }\end{array}$ & $\begin{array}{l}428 \\
396 \\
406 \\
407 \\
431 \\
388 \\
418 \\
405 \\
409 \cdot 8(14 \cdot 9)\end{array}$ & $\begin{array}{l}770 \\
903 \\
907 \\
886 \\
687 \\
973 \\
800 \\
787 \\
839 \cdot 1(93 \cdot 3)\end{array}$ & $\begin{array}{l}475 \\
438 \\
430 \\
432 \\
481 \\
422 \\
454 \\
436 \\
446 \cdot 0 \star(21 \cdot 8)\end{array}$ & $\begin{array}{l}750 \\
873 \\
770 \\
830 \\
653 \\
847 \\
913 \\
687 \\
790 \cdot 3+(91 \cdot 2)\end{array}$ & $\begin{array}{l}419 \\
398 \\
395 \\
408 \\
435 \\
395 \\
409 \\
397 \\
407 \cdot 0+(14 \cdot 1)\end{array}$ & $\begin{array}{r}1033 \\
1030 \\
917 \\
820 \\
800 \\
1043 \\
1183 \\
720 \\
944 \cdot 5+(156 \cdot 3)\end{array}$ \\
\hline
\end{tabular}

$\star p<0.001$ from baseline values.

†Not statistically different from baseline values. 
Della Bella, Grazi, Cipolla, Fabbiocchi, Rimondini, Sganzerla, Guazz

Table 3 Holter data in the study group

\begin{tabular}{|c|c|c|c|c|c|c|c|c|c|}
\hline \multirow[b]{2}{*}{ Patient No } & \multicolumn{3}{|c|}{ Heart rate $(\min / \max )$} & \multicolumn{3}{|c|}{ Total beats $/ 24 h$} & \multicolumn{3}{|c|}{ Extrasystoles $/ 24 h$} \\
\hline & $A$ & $\boldsymbol{B}$ & $C$ & $\boldsymbol{A}$ & $\boldsymbol{B}$ & $C$ & $A$ & $\boldsymbol{B}$ & $C$ \\
\hline $\begin{array}{l}1 \\
2 \\
3 \\
4 \\
5 \\
6 \\
7 \\
8\end{array}$ & $\begin{array}{l}55 / 105 \\
47 / 92 \\
54 / 100 \\
64 / 120 \\
60 / 104 \\
48 / 100 \\
50 / 90 \\
61 / 121\end{array}$ & $\begin{array}{l}57 / 105 \\
45 / 90 \\
58 / 105 \\
60 / 118 \\
62 / 100 \\
50 / 92 \\
48 / 92 \\
63 / 115\end{array}$ & $\begin{array}{l}54 / 110 \\
44 / 96 \\
60 / 105 \\
58 / 122 \\
55 / 106 \\
49 / 95 \\
47 / 85 \\
60 / 120\end{array}$ & $\begin{array}{r}104832 \\
89712 \\
102384 \\
111168 \\
107815 \\
84258 \\
100368 \\
120672\end{array}$ & $\begin{array}{r}106272 \\
83376 \\
110304 \\
112320 \\
105470 \\
86976 \\
101520 \\
119808\end{array}$ & $\begin{array}{r}108000 \\
91440 \\
101644 \\
114336 \\
106209 \\
87984 \\
97632 \\
115488\end{array}$ & $\begin{array}{r}12738 \\
416 \\
31 \\
0 \\
205 \\
17 \\
0 \\
75\end{array}$ & $\begin{array}{c}30284 \\
1285 \\
75^{\star} \\
0 \\
1013 \\
949 \dagger \\
0 \\
177\end{array}$ & $\begin{array}{r}14165 \\
375 \\
40 \\
0 \\
272 \\
85 \\
0 \\
82\end{array}$ \\
\hline
\end{tabular}

^Including 4 episodes of ventricular tachycardia (3-4 beats).

tIncluding 2 episodes of ventricular tachycardia ( 3 beats).

$A$, baseline recording; $B$, first 24 hours after pacing; $C$, recovery.

difference in the axis of the $\mathrm{T}$ wave, in the ventricular gradient, and QTc interval in the control group when we compared data collected before and after the electrophysiological study. Four patients had ventricular arrhythmias; but the frequency of spon- taneous arrhythmias seen during Holter monitoring after the electrophysiological study did not change In the four patients who did not have arrhythmias? during the baseline study there were no spontaneous ${ }_{1}$ arrhythmias during the second and third recordings $\frac{\mathbb{P}}{\mathcal{O}}$

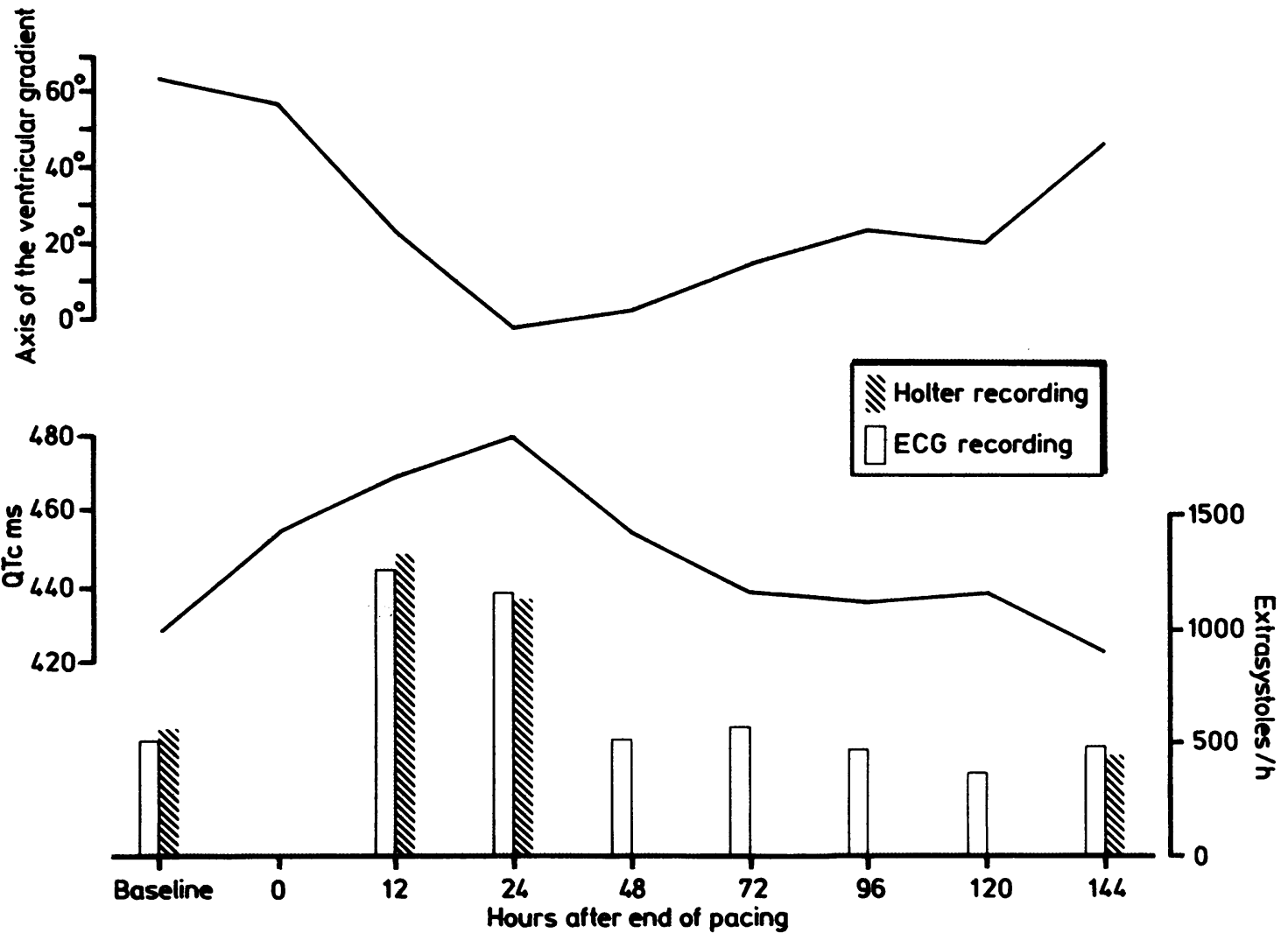

Fig 3 Axis of the ventricular gradient, QTc interval, and arrhythmia frequency expressed as ventricular extrasystoles per hour in the baseline recordings and after right ventricular pacing in patient 1 . The frequency of extrasystoles in the baseline recording and at 12, 24, and 144 hours (6th day) was obtained from Holter recordings. At other times data were obtained from direct electrocardiographic monitoring (see Methods). 


\section{Discussion}

The presence of changes in ventricular repolarisation and an increased frequency of spontaneous ventricular arrhythmias in the patients of the study group but not in the control group suggests that ventricular stimulation rather than other procedures was responsible for these changes.

\section{REPOLARISATION ABNORMALITIES INDUCED} BY VENTRICULAR PACING AND SPONTANEOUS ARRHYTHMIAS

Patients who had no arrhythmias in the baseline study did not show spontaneous arrhythmias when repolarisation abnormalities were induced by a 12 hour period of right ventricular pacing. In patients who presented with ventricular arrhythmias, however, we found that a 2-15 fold increase in ventricular arrhythmias and the presence of ventricular tachycardia were accompanied by the appearance of post-pacing repolarisation abnormalities. This, and the return to baseline values of the ventricular arrhythmias with recovery of normal repolarisation, virtually excluded spontaneous variability of ventricular ectopy. ${ }^{1314}$

The strict temporal relation between repolarisation abnormalities and worsening of spontaneous arrhythmias suggests that spontaneous electrical instability was exacerbated by changes in the ventricular gradient and the lengthening of the QTc interval. In some patients these repolarisation abnormalities may reflect an increased electrical instability.

The basis for this effect could be an increase in the inequalities of recovery in different areas of the myocardium. In a series of experiments by Kuo et al ${ }^{15}$ a previously "innocuous" premature stimulus could at a critical degree of dispersion of repolarisation trigger repetitive responses that eventually led to ventricular fibrillation. In that study changes in the $T$ wave and prolongation of QTc interval were evident at the time of induction of ventricular fibrillation. Also repetitive responses could be induced when a critical degree of dispersion of repolarisation was reached, even when spontaneous arrhythmias did not occur during the control period. These data suggest that electrical instability may be caused by repolarisation abnormalities even in the absence of spontaneous arrhythmias before the repolarisation abnormalities were induced.

The reported experimental data may be clinically relevant to our study. Our patients without arrhythmias in the baseline recording remained free from arrhythmias after pacing induced changes in repolarisation, probably because the degree of dispersion of repolarisation that was achieved was not sufficient to induce spontaneous arrhythmias. In contrast the increased electrical instability that accompanied the post-pacing repolarisation abnormalities in patients with pre-existing spontaneous ventricular arrhythmias may have increased the frequency of single ectopic complexes and the spontaneous occurrence of the repetitive arrhythmias.

Torsade de pointe has been described at the end of ventricular pacing (patient 1 in reference 16). The electrocardiogram of this patient showed inversion of the T wave in leads II, III, aVF, and in the precordial leads and no prolongation of the QT interval. These findings suggested a possible role of repolarisation abnormalities and increased electrical instability in the genesis of the arrhythmia.

\section{POSSIBLE MECHANISMS OF REPOLARISATION}

\section{ABNORMALITIES}

In all patients in this study right ventricular pacing was followed by a shift toward the left and upwards of the $T$ wave vector and of the ventricular gradient in the frontal plane. Our data show that the repolarisation sequence has shifted. Repolarisation is proceeding in the opposite direction to the basal state. This may be caused by a lengthening of action potential in the apical regions as a result of right ventricular apical pacing. Repolarisation proceeds from the epicardium to the endocardium ${ }^{1718}$; a positive $T$ wave is related to the shorter refractory period and shorter monophasic action potential in the epicardial than in the endocardial layers ${ }^{1920}$; the inversion of the $T$ wave in the precordial leads after ventricular pacing may reflect a relative lengthening of the action potential in the epicardium (or in the right surface of the interventricular septum) compared with the endocardial layers of the left ventricle.

The time course of changes and recovery to baseline values was similar for both ventricular gradient and QTc interval, suggesting that the two events may share a common mechanism. The lengthening of the QT interval could be related to the degree of dispersion of repolarisation caused by prolongation of action potential in the stimulation area.

\section{T WAVE MEMORY?}

It has been suggested that the persistence of the observed repolarisation abnormalities beyond the period of ventricular pacing is related to the possible effects of electrotonic currents. ${ }^{6}$ This view, however, has been criticised, ${ }^{212}$ because electrotonic currents, which are passive phenomena, should only affect the repolarisation sequence associated with a given depolarisation. Therefore, after the recovery of normal intraventricular activation a repolarisation sequence similar to the original should occur. 
Cohen et al proposed a different explanation for the development and persistence of the repolarisation abnormalities. ${ }^{23}$ They suggested that the continuous electrical activity of the heart itself is responsible for the genesis of an electrical gradient throughout the ventricles and that metabolic factors are involved in the maintenance of inequalities in repolarisation. The time course of recovery to normal repolarisation of our study suggests the possibility that metabolic factors can play a role in the persistence of the $T$ wave changes.

We found that the repolarisation abnormalities that occur after ventricular pacing, which previously have not be regarded as clinically relevant, may be clinically important. Inversion of the $T$ wave after ventricular pacing was accompanied by an increased electrical instability, which was manifested by a higher frequency of spontaneous ventricular arrhythmias. Patients in whom prolonged ventricular pacing is suddenly stopped may demonstrate increased electrical instability in the early phase after the end of pacing. Patients with pre-existing ventricular arrhythmias are particularly susceptible to such instability.

\section{References}

1 Denes P, Pick A, Miller RH, Pietras RJ, Rosen KM. A characteristic precordial repolarization abnormality with intermittent left bundle branch block. Ann Intern Med 1978;89:55-7.

2 Gould L, Venkataraman K, Goswami MK, Gomprecht RF. Pacemaker-induced electrocardiographic changes simulating myocardial infarction. Chest 1973;63:829-33.

3 Chatterjee K, Harris A, Davies G, Leatham A. Electrocardiographic changes subsequent to artificial ventricular depolarisation. Br Heart $\mathcal{f}$ 1969;31:770-9.

4 Nicolai P. Le syndrome de Wolff-Parkinson-White. In: Les cahiers de médicographie. Suresnes: Servier, 1966:35.

5 Nicolai P, Medvedowsky JL, Delaage M, Barnay C, Blache E, Pisapia A. Wolff-Parkinson-White syndrome: $T$ wave abnormalities during normal pathways conduction. $\mathcal{F}$ Electrocardiol 1981;14:295-300.

6 Rosenbaum MB, Blanco $\mathrm{HH}$, Elizari MV, Lazzari JO, Davidenko JM. Electrotonic modulation of the $\mathbf{T}$ wave and cardiac memory. Am $\mathcal{F}$ Cardiol 1982; 50:213-22.

7 Kuo CS, Amlie JP, Munakata K, Reddy CP, Surawicz B. Dispersion of monophasic action potential durations and activation times during atrial pacing, ventricular pacing, and ventricular premature stimu- lation in canine ventricles. Cardiovasc Res 1983; 17:152-61.

8 Avitall B, Levine HJ, Naimi S, Donahue RP, Pauker SG, Adam D. Local effects of electrical and mechan-ical stimulation on the recovery properties of the canine ventricle. Am $\mathcal{F}$ Cardiol 1982;50:263-70.

9 Urie P, Burgess MJ, Lux RL, Wyatt RF, Abildskov $\frac{\text { D }}{\sigma}$ JA. The electrocardiographic recognition of cardiac尺 states at high risk of ventricular arrhythmias. Circ Res 1978;42:350-8.

10 Burgess MJ. Relation of ventricular repolarization to. electrocardiographic $T$ wave-form and arrhythmia $\overrightarrow{\vec{\omega}}$ vulnerability. Am f Physiol 1979;236:H391-402.

11 Ashman R, Byer E. The normal human ventricular gradient. I. Factors which affect its direction and its relation to the mean $Q R S$ axis. Am Heart $\mathcal{F} Y$ 1943;25:16-35. 12 Bazett HC. An analysis of the time relations of electro- $\vec{\infty}$
cardiogram. Heart 1920;7:353-70.

13 Winkle RA. Antiarrhythmic drug effect mimicked by윽 spontaneous variability of ventricular ectopy. Circu- lation 1978;57:1116-20.

14 Morganroth J, Michelson EL, Horowitz LN, 음 Josephson ME, Pearlman AS, Dunkman WB. Limitations of routine long term electrocardiographic monitoring to assess ventricular ectopic frequency. $\vec{\theta}$ Circulation 1978;58:408-14.

15 Kuo CS, Munakata K, Reddy CP, Surawicz B. Charac-.. teristics and possible mechanisms of ventricular arrhythmia dependent on the dispersion of action potential durations. Circulation 1983;67:1356-67.

16 Steinbrecher UP, Fitchett DH. Torsade de pointes. A cause of syncope with atrioventricular block. Arch $\frac{\mathrm{Q}}{\mathrm{Q}}$ Intern Med 1980;140:1223-6.

17 Spach M, Barr RC. Ventricular intramural and epicar- $\overrightarrow{\overrightarrow{0}}$ dial potential distributions during ventricular activa- 3 tion and repolarisation in the intact heart. Circ Res? 1975;37:243-57.

18 Moore EN, Preston JB, Moe GK. Durations of transmembrane action potentials and functional refractory periods of canine false tendon and ventricular myocardium. Circ Res 1965;17:259-73.

19 Reynolds EW, Vander Ark CR. An experimental study of the origin of $T$ waves based on determinations of $\delta$ effect refractory period from epicardial and endo- 3 cardial aspects of the ventricle. Circ Res 1959; 음 8:943-9.

20 Higuchi $T$, Nakaja $Y$. T wave polarity related to the $\frac{D}{O}$ repolarization process of epicardial and endocardial ventricular surfaces. Am Heart $\mathcal{F}$ 1984;108:290-5. N

21 Hoffman BF. Electrotonic modulation of the $T$ wave. Am F Cardiol 1982;50:361-2.

22 Surawicz B. Transient $\mathrm{T}$ wave abnormalities in inter- $\mathbb{\omega}$ mittent bundle branch block. Am $\mathcal{f}$ Cardiolo 1982;50:363-4.

23 Cohen I, Giles W, Noble D. Cellular basis for the T wave of the electrocardiogram. Nature 1976;262: 657-61. 